\title{
Impact of Operations Research on Computer Science
}

\author{
Mobin Ahmad \\ Department of Mathematics, Faculty of Science, Jazan University, Jazan, Saudi Arabia.
}

\begin{abstract}
Operations research on computers interact in numerous logical fields of imperative significance to our society. These incorporate, among others, transportation, financial matters, speculation procedure, stock control, co ordinations, wellbeing, dependability, urban arranging, and ecology. Computers and Operations Research (COR) gives a global discussion to the application of computers and operations research techniques to issues in these and related fields. The field of mathematics plays essential part in various fields. One of the important areas in mathematics is Operations research which is utilized as a part of basic models. This basic course of action of different articles or technologies prompt new innovations and adjustments in the current condition for upgrade in those fields. This paper gives a review of the utilizations of Operations research in heterogeneous fields to some degree yet predominantly concentrates on the computer science application that utilizes Operations research ideas. Various papers in light of Operations research have been concentrated identified with booking ideas, computer science applications and an outline has been presented here.
\end{abstract}

Keywords: Operations research, computer science, importance, society, application, techniques, problems, mathematics, models.

\section{Introduction}

First operations research models for upkeep improvement of computer science were created in the sixties by pioneers like Barlow, Prochain, Jorgenson and McCall. Fundamentally, a support improvement model is a mathematical model in which both expenses and advantages of upkeep are evaluated and in which an ideal harmony between both is acquired. The range ended up being productive for researchers. Operations research thoughts are profoundly used by computer science applications. Particularly in research regions of computer science such data mining, image segmentation, grouping, image capturing, networking etc., For example a data structure can be outlined as tree which thus used vertices and edges. So also demonstrating of network topologies should be possible utilizing Operations research. Similarly the most important concept of Operations research is used in asset designation, planning. Additionally, ways, strolls and circuits in Operations research are utilized as a part of huge applications say voyaging businessperson issue, database plan ideas, resource networking. This prompts the improvement of new algorithms and new hypotheses that can be utilized as a part of tremendous applications.

In the years quickly taking after the finish of World War II, O.R. developed quickly the same number of researchers understood that the rule that they had connected to solve problems for the military were similarly pertinent to many problems in the regular citizen division. These extended from here and now issues, for example, booking and stock control to long haul issues, for example, vital arranging and asset designation. George Dantzig, who in 1947 developed the simplex algorithm for Linear Programming (LP), gave the absolute most vital forces for this growth. Right up 'til today, LP stays a standout amongst the most generally utilized of all O.R. techniques and notwithstanding the generally late improvement of inside point strategies as an alternative approach, the simplex calculation (with various computational refinements) keeps on being broadly utilized. The second significant stimulus for the growth of O.R. was the quick advancement of digital computers throughout the following three decades. The basic technique was executed on a computer for the first time in 1950, and by 1960 such usage could solve problems with around 1000 imperatives. Today, implementations on powerful workstations can routinely solve problems with countless factors and constraints. Also, the huge volumes of information required for such issues can be put away and controlled very efficiently.

\section{Review of literature:}

Once the simple method had been imagined and utilized, the development of different strategies taken after at a fast pace. The next twenty years saw the development of the majority of the O.R. techniques that are being used today including nonlinear, integer and dynamic programming, computer simulation, PERT/CPM, queuing theory, inventory models, game theory, and sequencing and scheduling algorithms. The researchers who built up these strategies originated from many fields, most notably mathematics, engineering and economics. It is fascinating that the hypothetical bases for huge numbers of these techniques had been known for a considerable length of time, e.g., the EOQ recipe utilized with many stock models was created in 1915 by Harris, and a large portion of the lining formulae were produced by Erlang in 1917. Be that as it may, the period 
from 1950 to 1970 was the point at which these were formally brought together into what is viewed as the standard toolbox for an operations research expert and successfully applied to problems of mechanical criticalness. The accompanying area depicts the approach taken by operations research with a specific end goal to solve problems and investigates how these techniques fit into the O.R. framework.

One of the real main impetuses behind the growth of O.R. has been the rapid growth in computer technology and the concurrent growth in data frameworks and mechanized data storage and recovery. This has been an extraordinary aid, in that O.R. analysts now have prepared get to to data that was already difficult to get. All the while, this has likewise made things troublesome in light of the fact that many organizations wind up in the circumstance of being information rich however data poor. As it were, despite the fact that the data is all present "somewhere" and in "some form," removing valuable data from these sources is frequently extremely troublesome. This is one reason why information systems experts are precious to groups required in any nontrivial O.R. project. Data collection can importantly affect the past stride of issue definition and additionally on the accompanying stride of model formulation.

Data Collection: O.R. process data is gathered with the goal of interpreting the problem defined in the second stage into a model that can then be equitably investigated. Data typically originates from two sources perception and gauges. The main compares to the situation where data is really gathered by observing the system in operation and normally, this information has a tendency to get from the innovation of the framework. For example, operation times may be acquired by time studies or work strategies examination, asset utilization or scrap rates may be gotten by making test estimations over some reasonable interim of time, and data on requests and accessibility may originate from deals records, buy requests and stock databases. Other data are acquired by utilizing measures; a considerable measure of cost related data tends to fall into this class. For example, most organizations have standard esteems for cost things, for example, time-based compensation rates, stock holding charges, offering costs, and so on.; these guidelines should then be combined properly to compute costs of different exercises. Once in a while, information may likewise be requested explicitly for the current issue using reviews, polls or different psychometric instruments.

Computer Simulation Models: With the growth in computational power these models have turned out to be to a great degree mainstream in the course of the last ten to fifteen years. A simulation model is one where the system is dreamy into a computer program. While the particular computer language utilized is not a characterizing trademark, a number of languages and software systems have been produced exclusively with the end goal of building computer simulation models; an overview of the most famous frameworks might be found in OR/MS Today. Typically, such software has language structure and additionally implicit develops that take into consideration simple model development. All the time they additionally have arrangements for graphics and animation that can help one imagine the system being simulated. Simulation models are investigated by running the software over some timeframe that speaks to a reasonable period when the original system is working under unfaltering state. The inputs to such models are the choice factors that are under the control of the chief. These are dealt with as parameters and the recreation is keep running for different blends of qualities for these parameters. Toward the finish of a run statistics are accumulated on different measures of execution and these are then broke down utilizing standard techniques. The chief then chooses the mix of qualities for the choice factors that yields the most desirable performance.

Simulation models are to a great degree powerful and have one exceptionally alluring element: they can be utilized to display extremely complex systems without the need to make excessively numerous improving suspicions and without the need to give up detail. Then again, one must be exceptionally cautious with simulation models in light of the fact that it is likewise simple to misuse simulation. To begin with, before utilizing the model it must be legitimately approved. While approval is fundamental with any model, it is particularly imperative with simulation. Second, the expert must be comfortable with how to utilize a simulation model accurately, including things, for example, replication, run length, warm-up and so forth; a point by point clarification of these ideas is past the extent of thisstudy however the intrigued perused ought to allude to a decent content on simulation. Third, the investigator must be comfortable with different statistical techniques keeping in mind the end goal to dissect simulation output in an important manner. Fourth, building a complex simulation model on a computer can regularly be a testing and moderately tedious undertaking, despite the fact that simulation software has created to the point where this is getting to be plainly less demanding by the day. The reason these issues are underscored here is that a current simulation model can be exceptionally garish and alluring, however its genuine esteem lies in its capacity to yield experiences into extremely complex problems. Be that as it may, with a specific end goal to get such experiences an extensive level of specialized expertise is required. 
Analogic Models: These are models that are a stage down from the first category in that they are physical models too; however utilize a physical analog to portray the system, rather than a correct downsized variant. Maybe the most popular case of an analogic model was the ANTIAC model (the acronym stood for hostile to programmed calculation) which exhibited that one could lead a legitimate operations research examination without falling back on the utilization of a computer. In this problem the goal was to locate the most ideal approach to convey supplies at a military stop to different request focuses. Such an issue can be fathomed proficiently by utilizing strategies from network flow analysis. However the real technique that was utilized took a different approach. An ant colony dwelling place on a raised stage was picked as a simple for the warehouse and little hills of sugar all alone stages were spoken to each request point. The network of streets associating the different hubs was developed utilizing bits of string with the length of each being relative to the genuine separation and the width to the limit along that connection. A multitude of ants was then discharged at the ant colony dwelling place and the ways that they got to the hills of sugar were then watched. After the model achieved an enduring state, it was found that the ants by righteousness of their own propensities had found the most productive ways to their goals! One could even lead some post optimality analysis. For example, different transportation limits along each connection could be broke down by proportionately shifting the width of the connection, and a situation where certain streets were unusable could be examined by essentially evacuating the relating connections to perceive what the ants would then do. This delineates an analogic model. All the more critically, it additionally represents that while O.R. is commonly related to mathematical analysis, the utilization of an inventive model and critical thinking strategy, for example, the one simply depicted is a completely real approach to direct an O.R. study.

Model Solution: The fifth phase of the O.R. process is the solution of the problem represented by the model. This is the area on which a tremendous sum of research and improvement in O.R. has been engaged, and there is a plenty of techniques for breaking down a wide range of models. It is difficult to dive into points of interest of these different techniques in a solitary introductory study, for example, this; notwithstanding, a diagram of a portion of the more important methods can be discovered somewhere else in this handbook. As a rule, some formal preparing in operations research is essential keeping in mind the end goal to acknowledge what number of these techniques work and the intrigued perused is asked to scrutinize an initial content on O.R.; the area on "Further Reading" toward the finish of the study records some great books. It is likewise worth specifying that as of late various software systems have risen which (from a certain point of view) "secret elements" for understanding different models are. Be that as it may, some formal instruction in O.R. methods is as yet required (or if nothing else emphatically prescribed) before utilizing such systems. From the point of view of the expert, the most important thing is to have the capacity to perceive which of the numerous available techniques is fitting for the model developed. For the most part, this is not a hard assignment for somebody with some simple preparing in operations research. The techniques themselves fall into a few classifications. At the most reduced level one may have the capacity to utilize basic graphical techniques or even experimentation. Notwithstanding, in spite of the way that the advancement of spreadsheets has made this substantially less demanding to do, it is normally an infeasible approach for generally nontrivial problems. Most O.R. techniques are investigative in nature, and can be categorized as one of four broad categories. In the first place, there are reproduction techniques, which clearly are utilized to investigate simulation models. A critical piece of these are the real computer programs that run the model and the strategies used to do as such effectively.

At this point, a few words about computational aspects are all together. At the point when connected to a nontrivial, genuine issue the greater part of the techniques discussed in this area require the utilization of a computer. In reality, the single greatest driving force for the expanded utilization of O.R. methods has been the fast increment in computational power. In spite of the fact that there are still vast scale issues whose arrangement requires the utilization of mainframe computers or effective workstations, numerous enormous problems today are fit for being tackled on desktop microcomputer systems. There are numerous computer packages (and their number is developing by the day) that have turned out to be prevalent as a result of their convenience and that are ordinarily accessible in different forms or sizes and interface consistently with different software systems; contingent upon their particular needs end-clients can choose a proper setup. A large portion of the software vendors additionally offer preparing and counseling administrations to bail clients with getting the most out of the systems. Some specific techniques for which business software implementations are accessible today incorporate enhancement/mathematical programming (counting linear, nonlinear, integer, dynamic and goal programming), network flows, simulation, statistical analysis, queuing, forecasting, neural networks, decision analysis, and PERT/CPM. Additionally available today are business software systems that join different O.R. techniques to address particular application territories including transportation and co ordinations, generation arranging, stock control, planning, area examination, determining, and inventory network administration. A few cases of prominent O.R. software systems incorporate CPLEX, LINDO, OSL, MPL, SAS, and SIMAN, to give some examples. While it would unmistakably be difficult to depict in this the 
components of all accessible software, magazine, for example, OR/MS Today and IE Solutions routinely distribute isolate overviews of different classifications of software systems and bundles. These productions likewise give pointers to various sorts of software available; for instance, the December 1997 issue of OR/MS Today (pages 61-75) gives a total asset registry to software and specialists. Updates to such registries are given occasionally. The primary point here is that the capacity to understand complex models/issues is far less of an issue today than it was 10 years or two prior, and there are a lot of promptly accessible assets to address this issue.

Applications of Operations Research: Operations Research ideas are broadly used to study and model various applications, in computer science regions. Essentially, Operations Research is utilized as a part of human science for instance to gauge on-screen character's notoriety or to investigate dissemination instruments. Operations Research is utilized as a part of science and preservation endeavors where a vertex speaks to areas where certain species exist and the edges speak to relocation way or development between the locales. This data is vital when taking a gander at reproducing examples or following the spread of illness, parasites and to concentrate the effect of movement that influences different species. Computer science ideas are generally utilized as a part of Operations Research. For instance, the voyaging salesman problem, the most limited spreading over tree in a weighted chart, acquiring an ideal match of occupations and men and finding the briefest way between two vertices in a diagram. It is likewise utilized as a part of displaying transport networks, activity networks and theory of games. The network activity is utilized to take care of huge number of combinatorial issues. The most well known and successful applications of networks in OR is the arranging and planning of huge convoluted undertakings. The best well known problems are PERT (Project Evaluation Review Technique) and CPM (Critical Path Method). Next, Game theory is connected to the issues in designing, financial aspects and war science to discover ideal approach to play out specific assignments in aggressive situations. To speak to the technique for finite game a digraph is utilized. Here, the vertices speak to the positions and the edges speak to the moves.

\section{Conclusion}

The main aim of this paper is to present the importance of Operations Research thoughts in different zones of compute applications for researches that they can utilize Operations Research ideas for the exploration. A diagram is exhibited particularly to extend the possibility of Operations Research. Thus, the Operations Research area of this paper is given significance than to alternate areas. Inquires about may get some data identified with Operations Research and its applications in computer field and can get a few thoughts identified with their field of research.

\section{References}

[1]. Adam Schenker, Mark Last, horst Banke,Abraham andel,"Clustering of Web documents using a Operations Research", Springer werlog, Septermber 2007.

[2]. Anindya J.Pal, Samar S.Sarma, Biman Ray, "CCTP, Operations Research algorithms - Soft computing Solutions IEEE, 2007

[3]. Bing Hong Liu, Wel Chieh Ke, Chin-Hsien Tsai, Ming-Jer Tsai, "Constructing a message pruning tree with minimum cost for tracking moving objects in wireless sensor networks", IEEE Volume 57, Number 6, July 2008

[4]. Daniel Marx, "Operations Research problems and their applications in scheduling",

[5]. Gian Luca Marcialis, Fabio Roli, Alessandra Serrau, "Operations Research Based and Structural Methods for Fingerprint Classification, Springer verlag, Berlin Heidelberg 2007

[6]. John.P.Hayes, “A Operations Research Model for Fault Tolerant Computing Systems”, IEEE September 1976

[7]. Narasingh Deo, “Operations Research with applications to engineering and computer science”, Prentice Hall of India, 1990

[8]. Perri Mehonen, Janne Riihijarvi, Marina Petrova, "Automatic Channel allocation for small wireless area networks using Operations Research algorithm approach", IEEE 2004

[9]. Shariefuddin Pirzada and Ashay Dharwadker, "Journal of the Korean Society for Industrial and applied Mathematics, Volume 11, No.4, 2007

[10]. Sven Dickinson, Pelillo, Ramin Zabih, "Introduction to the special section on Operations Research algorithms in computer vision", IEEE on pattern analysis, Vol 23 No. 10, September 2001

[11]. V.P.Eswaramoorthy, "New algorithm for analyzing performance of neighbourhood strategies in solving job shop scheduling problems, Journal of Scientific \& Industrial Research, August 2008

Mobin Ahmad. "Impact of Operations Research on Computer Science." IOSR Journal of Computer Engineering (IOSR-JCE) 19.4 (2017): 50-53. 